\title{
Novel Synthesis and Characterization of Vegetable Oil Derived Corrosion Inhibitors
}

\author{
S. Kasshanna, P. Rostron* \\ The Petroleum Institute, Umm Al Nar, Main Campus, Dept. of Applied Chemistry,2533 Abu Dhabi, United Arab Emirates
}

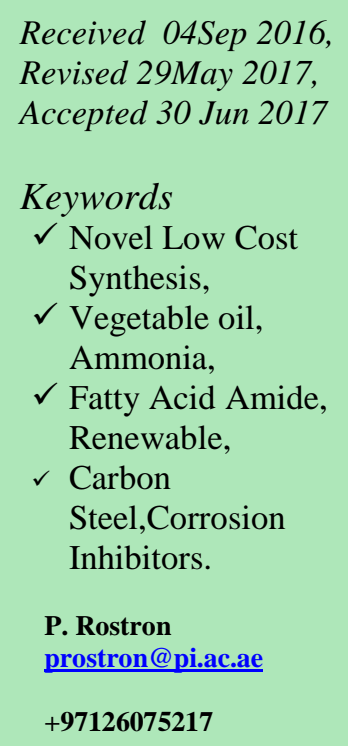

\begin{abstract}
This paper presents a novel one-pot synthesis of fatty acid amides, derived from natural vegetable oils. A series of vegetable oils, which includes: linseed oil, sunflower oil, peanut oil, cress oil, almond oil and canola oil, were esterified via transesterification reaction. This first step reaction was then followed by addition of ammonia $\left(\mathrm{NH}_{3}\right)$ under reflux conditions to produce the corresponding fatty acid amide. The progress of the reaction was followed qualitatively using Thin Layer Chromatography (TLC) technique with ethyl acetate as eluent. Fourier Transform Infrared Spectroscopy (FTIR) was used to investigate the different samples of the reaction mixture in order to determine the functional group transformation. In this method, no cleanup between steps was attempted. The idea is to try to produce effective corrosion inhibitors at very low cost. Corrosion studies were performed on the product from the reaction, without cleanup or isolation, again to reduce commercial cost. Corrosion studies of carbon steel were performed in vigorously stirred aerated seawater, one of the most corrosive natural environments. Corrosion rate measurements were recorded to identify the corrosion rate by mass loss over a specific time period (one week). LPr was recorded by Gamry potentiostat and mass loss recorded using a $0.1 \mathrm{mg}$ analytical balance. Our results showed correlation between the TLC and FTIR techniques indicating a successful progress of the reaction and functional group transformation. In addition, the corrosion inhibition efficiencies obtained using the corrosion measurement methods were up to $98 \%$ for some of the oil samples, equivalent to a corrosion rate reduction of 44 . The one pot synthetic route chosen is a low cost approach to the formation of fatty acid amides.
\end{abstract}

\section{Introduction}

Corrosion is commonly known as "rusting, an undesirable phenomenon which destroys the luster and beauty of objects and shortens their lives" [1]. It is defined as an electrochemical reaction between a material whether it is a biomaterial, nanomaterial, a polymer, or a metal and its environment $[2,3]$. The cost of corrosion has been assessed in several studies and usually lies $n$ arrange of between $3-6 \%$ of GDP.

Studies of the cost of corrosion have not been performed in the UAE, but it is reasonable to assume it lies within the range observed in other countries and one can therefore assume that for the UAE, with a GDP of AED 953.2 Billion in 2013, the annual cost of corrosion lies in the range 28.6 - 57.2 billion dirhams. Most of these studies on the cost of corrosion have also demonstrated that that between $20-40 \%$ of the cost is preventable, using current technology. It is therefore possible to show that the UAE economy as a whole could obtain a cost saving of between 6.0 -22.9 Billion Dirhams per year if corrosion was under better control. [4, 5, 6].

Carbon steel is an alloy mixture generally made of carbon and iron. Carbon steel has become a preeminent construction and fabrication material for the modern society, especially for petroleum pipelines. It has high strength, ease of machining and fabrication, ductility, weldability and susceptibility for heat treatment for varying mechanical properties. All these properties, together with relatively low cost, are the main reason for its popularity and its applicability in different industrial areas [7, 8]. Nevertheless, this material has a significant drawback: in many of the natural environments it will react with the environment and undergo spontaneous decomposition to a non-metallic form [9].

Research has identified four different approaches to control the corrosion of carbon steel: prevention methods identified as (1) coatings, (2) cathodic protection, (3) material selection, and (4) corrosion inhibitors. Coatings are materials applied to the metal to separate its contact with corrosive environments. However, coatings fail over time and need to be reapplied. In general the dry film thickness determines coatings life [8, 10, 11]. Material selection is the ability to select an appropriate alloy to match the conditions expected during the service life. 
Cathodic protection is an electrochemical process in which an electric current flows onto the metal surface, decreasing the voltage to protect the metal surface against corrosion. The key problems with cathodic protection are that the material must be submerged in an electrolyte and the driving voltage continually adjusted to provide the correct amount of voltage lowering, too low and the potential for hydrogen damage can occur $[4,12]$.

Corrosion inhibitors are organic or inorganic chemicals that interfere with the corrosion process to protect against the internal corrosion. The chemicals can be added via several different processes, either continual injection or batch (periodic injection).[2, 13]. For pre-existing pipelines, inhibitors are the main approach to reduce corrosion rates as they can be injected into the flow to control corrosion as needed.

This research has focused on the formation of corrosion inhibitors from vegetable oils as they are cheap and ubiquitous and biodegradable.in our experiments, different vegetable oils were refluxed with sodium methoxide to produce the corresponding methylester, then ammonia was added to the mixture and was refluxed at specific reaction conditions. The purpose is to synthesize using a single-pot, renewable and cheap fatty acid amide corrosion inhibitor. Vegetable oils are renewable, natural, available and low cost products that have many interesting properties such as their biodegradability and non-toxicity, their chemical composition, their degree of unsaturation and the position and stereochemistry of their double bonds in their chains [14]. They are used as renewable raw materials for many industrial processes such as paint formulation for example [15].

Generally, vegetable oils chemical composition consists of 90 to $98 \%$ of triglycerides which are esters containing different fatty acids including oleic, linoleic acid, alpha linoleic, stearic, palmitic and eicosanoic acid $[16,17,18]$. Previous research work has indicated that the high electron density of an amide group will promote attraction to the electropositive surface of a metal, such as carbon steel [19]. The long hydrocarbon chains then de-water the metal surface and prevent water reaching the metal surface, thus reducing corrosion.The advantage of our study is that by conducting the reaction in a one pot synthesis without cleanup, the synthesis costs are reduced significantly.

\section{Literature review}

The synthetic route to the formation of the fatty acid amide has been examined and implemented by many researchers for many application purposes and was reported as two-steps in which the first is the transesterification of vegetable oil to produce methylester followed by the addition of amine to produce fatty acid amide.The synthesis of fatty amide polymers was reported by Sujata Pramanik et al. At first sodium methoxide was prepared using excess of dry methanol. Then castor oil, was added and the mixture was refluxed for 30 minutes at $150{ }^{\circ} \mathrm{C}$. Diethanolamine was added with $10 \mathrm{~mol} \%$ excess to the methylester formed and refluxed for one and a half hour with continuous stirring under $\mathrm{N}_{2}$ gas atmosphere [20]. Thus, the synthesis described is however beyond the purpose of our study. According to the paper published by El Kadi et al., the synthesis of neem oil ester was described by the formation of methylester in sulfuric acid-catalyzed $\left(\mathrm{H}_{2} \mathrm{SO}_{4}\right)$ esterification with methanol followed by a base-catalyzed $(\mathrm{NaOH}, \mathrm{KOH})$ conversion with methanol under certain conditions. The reason of the two-steps reactions is to eradicate the free fatty acids in the oil to ensure the formation of biodiesel and glycerol [21]. A different method of amide synthesis was implemented in a research study published by O.A. Anyebe et al. in which ammonium salt and methylamine replaced ammonia. $3.84 \mathrm{~g}$ of ground oil were heated to $50{ }^{\circ} \mathrm{C}$ and then mixed with $15 \mathrm{~mL}$ of acetone in a round-bottom flask. $0.5 \mathrm{~g}$ of sodium hydroxide was added to the mixture and temperature was maintained for 10 minutes. Ammonium Chloride (salt) was added in situ with constant stirring until all the water was excluded. Once the product desired was formed, $70 \mathrm{~mL}$ of $0.5 \mathrm{~mL}$ aqueous $\mathrm{NaCl}$ was added to the mixture to wash out the solid product and ensure a complete reaction of $\mathrm{NaOH}$, ammonium chloride and glycerol. Acetone was then added to wash and dry the fatty amide formed at $35^{\circ} \mathrm{C}$. Acetone was removed by evaporation. Methylamine was added to react with the oil following the same steps. The products formed in both reactions were (1) [oil $+\mathrm{NH}_{4} \mathrm{Cl}$ product] and (2) [oil $\left.+\mathrm{CH}_{3} \mathrm{NH}_{2}\right]$ with same solubility properties [22].

\section{Mechanism of the reaction}

The mechanisms of the fatty acid amide synthesis is well understood and described in a two-step reaction and are shown in Figures 1 and 2 below. The general mechanism of transesterification begins with a pre-step of deprotonation of the methanol by sodium metal to form a stronger nucleophilic species; sodium methoxide. The deprotonation is then followed by a nucleophilic attack of the methoxide ion to the carbonyl group to form a tetrahedral alkoxide intermediate. At that juncture, the restoration of the carbonyl group leads to the formation of a diglyceride and release of a methylester. Finally, the methanol loses its proton and produces a new sodium methoxide ion. These two steps are repeated three times to produce respectively the diglyceride alkoxide, and at that point, the monoglyceride fatty acid alkoxide and eventually releases the glycerol[21, 23]. 


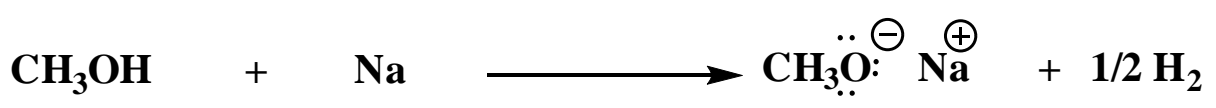

\section{Step 1: $\underline{\text { Transesterififcation }}$}
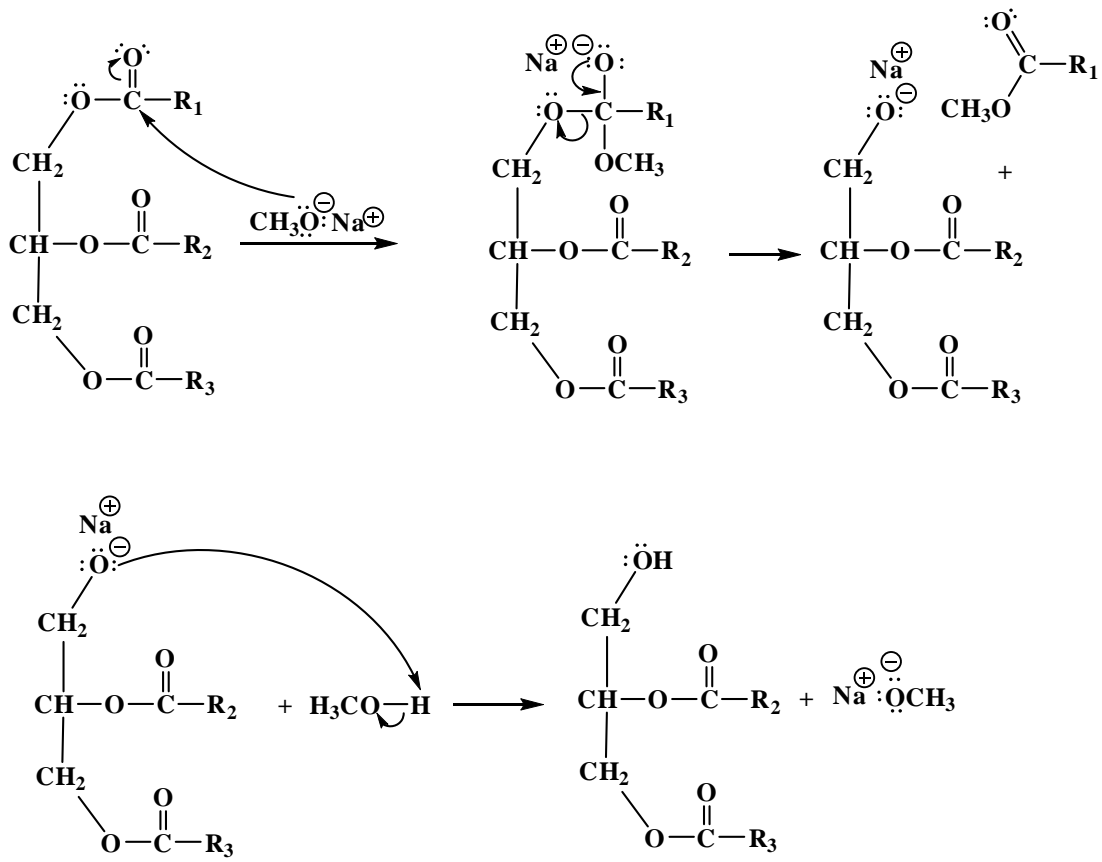

Fig2:Transesterification mechanism [23]

\section{Step 2: The General Mechanism for Amide Formation}

The mechanism of the fatty acid amide formation consists of a nucleophilic attack of the nitrogen in ammonia $\left(\mathrm{NH}_{3}\right)$ onto the carbon in the carbonyl group of the methylester. This step leads to the formation of a tetrahedral alkoxide intermediate, followed by proton transfer between the oxygen in the alkoxide ion and the ammonium ion. Finally, the elimination of the alkoxide ion takes place leading to the release of the methanol and the formation of the amide[17]:

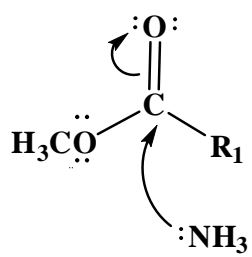

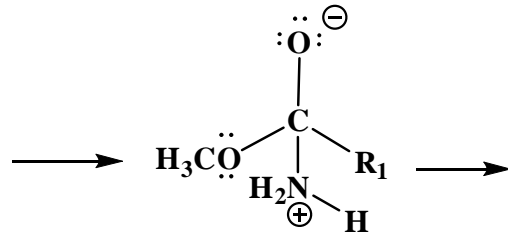

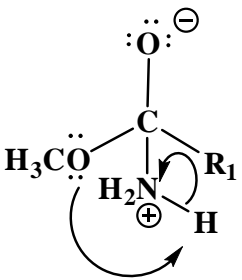<smiles>C[O+]=N[Mg]</smiles>

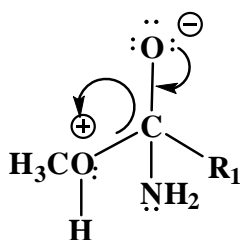

Fig. 2: The mechanism of the formation of fatty acid amide [23] 


\section{Experimental}

\subsection{Materials}

The vegetable oils were standard commercial food grade oils, within their shelf life. The oils are classified according to vegetative source: almond oil, cress seed oil, linseed oil, peanut oil, sunflower oil and canola oil. Reagents were supplied from BDH (UK) either reagent or analytical grades: sodium metal (Na) was supplied from $\mathrm{BDH}$ reagent grade, methanol $\left(\mathrm{CH}_{3} \mathrm{OH}\right)$ and ethyl acetate solvents were supplied from BDH ANALAR grade, concentrated aqueous ammonia, (sp.gr $0.880 \mathrm{gmL}^{-1}$ ).

\subsection{Experimental procedure}

Step 1

A $250 \mathrm{~mL} 3$ necked round bottomed flask equipped with magnetic stirrer bar, reflux condenser, dry gas inlet and stopper was baked at $140^{\circ} \mathrm{C}$ for 6 hours to ensure complete dryness and was assembled hot over a hotplate stirrer and allowed to cool under dry nitrogen.

To the cooled flask was added $00 \mathrm{~mL}$ anhydrous methanol (dried over anhydrous magnesium sulfate overnight) followed by $0.060 \mathrm{~g}$ clean and dry sodium metal. The mixture was stirred under dry nitrogen until all of the metal had dissolved, followed by the addition of $20 \mathrm{~mL}$ of vegetable oil. The mixture was stirred vigorously and heated to reflux via external oil bath and hotplate stirrer. The reaction was followed by periodic TLC and in general, the reaction was found to be complete in approximately one hour.

Step 2

The temperature was increased to $150^{\circ} \mathrm{C}$ and small periodic aliquots $(3 \mathrm{x} 3 \mathrm{~mL})$ of concentrated aqueous ammonia added per hour. The reaction was followed by TLC and via FTIR. When the reaction was judged to be complete the mixture was cooled and bottled.

Figure 3 below shows a picture of the glassware setup taken during the progress of the reaction.

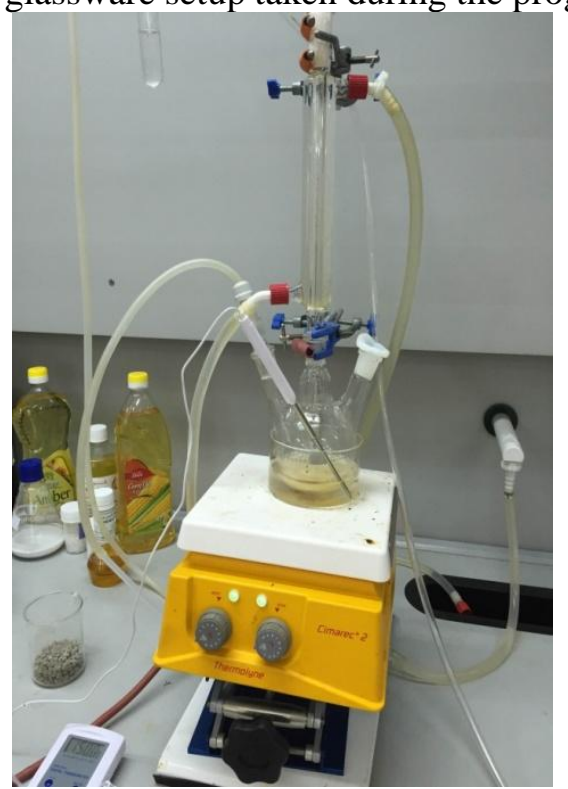

Fig. 3: Apparatus to form fatty acid amide.

\subsection{Characterization Techniques}

TLC and FTIR techniques were used respectively to constantly monitor the progress of the reaction and the functional group transformation. Additionally, the corrosion rate variation and the corrosion inhibition efficiency of the product formed were investigated using the LPR and mass loss techniques.

\subsubsection{Thin Layer Chromatograph (TLC)}

Spots of each of the oil, the methylester and the fatty acid amide were placed on a TLC plate and eluted with ethyl acetate solvent. The difference in the retention time of the different spots is a good indicator of the progress of the reaction and the formation of a new product. The spots of oil, methylester and amide were compared on a shortwave UV lamp 


\subsubsection{Fourier Transform Infrared FTIR}

The functional groups transformation of the oil, the methylester and the fatty acid amide were followed using FTIR. The carbonyl stretch in a methyl ester has a characteristic strong absorption at $1750-1745 \mathrm{~cm}^{-1}$. The amide stretch however has a characteristic absorption at $1630-1690 \mathrm{~cm}^{-1}$. This significant difference is easily seen and is a good measure of the formation of the amide bond.

\subsubsection{Mass Loss Measurement}

Samples of carbon steel plate were suspended in seawater for one week containing different inhibitor concentrations, in order to prove the long term stability of the inhibitors synthesized. The effect of concentration was measured, with an optimized effective inhibitor concentration of $1000 \mathrm{ppm}$.

\subsubsection{Linear Polarization Resistance (LPR)}

$150 \mathrm{~mL}$ flat beaker was equipped with 1 carbon counter anode, a sample metal holder, a reference electrode and an aerator. The flask was filled with $100 \mathrm{~mL}$ of fresh seawater. Coupons of carbon steel disks of approximately $25 \mathrm{~mm}$ diameter and $10 \mathrm{~mm}$ thickness were prepared by cutting them from round bar standard mild steel to use as metal samples. The electrode was connected to the disk via hole drilled onto the edge. The wire was electrically bonded to the coupon using molten solder and the connection isolated from the solution by filling the hole with epoxy resin as per the procedure published by Rostron [5].

\section{Results and Discussion}

A correlation between the results obtained by TLC and FTIR was shown and described below. The LPR and mass loss techniques were used to check the corrosion inhibition efficiency. Almost all of the samples showed high corrosion efficiencies from 85 to $98 \%$ except for the canola amide.

\subsection{Thin Layer Chromatography}

Figures 4 to 8 shows the TLC plates of the different vegetable oil investigated. The difference in the retention time between the starting material (vegetable oil), the methylester and the amide formed is clear and indicates the progress of the reaction.

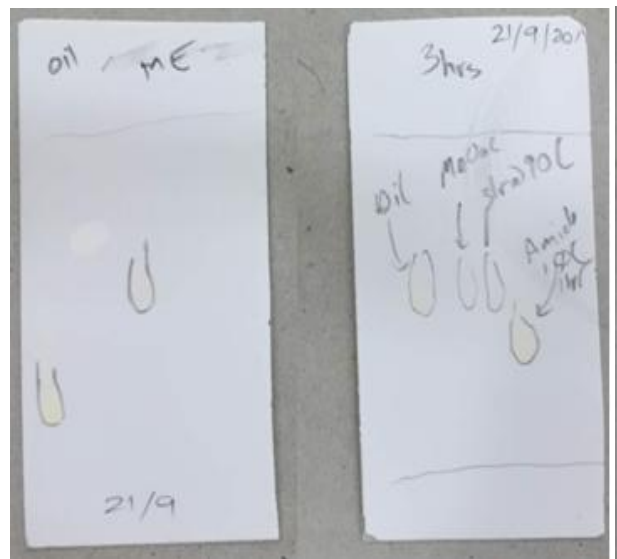

Fig. 4: TLC plates of linseed sample

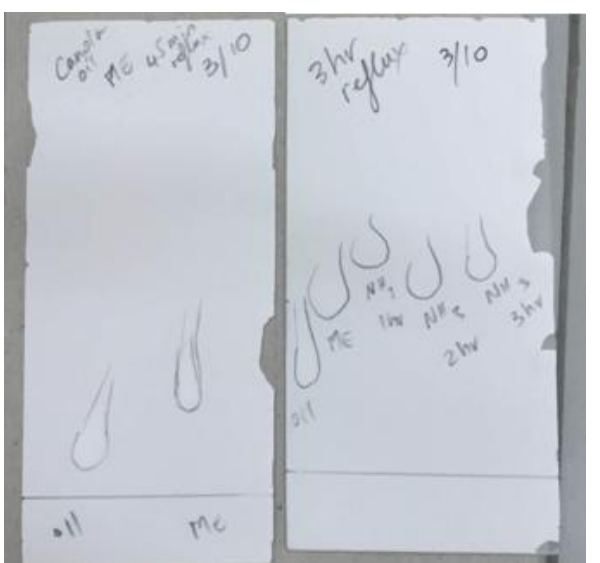

Fig. 5: TLC plates of canola sample

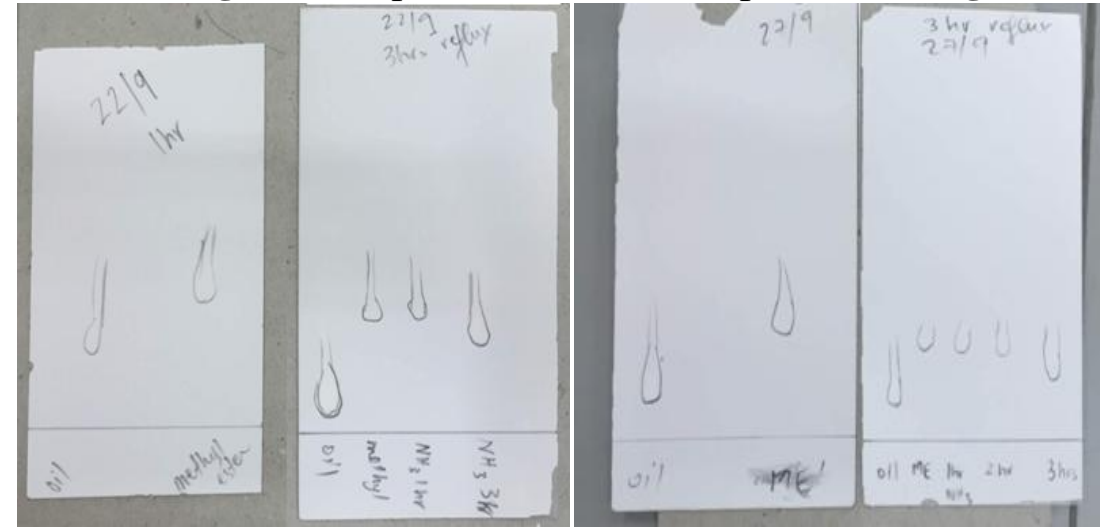

Fig. 6: TLC plates of sample
Fig. 7: TLC plates of sample

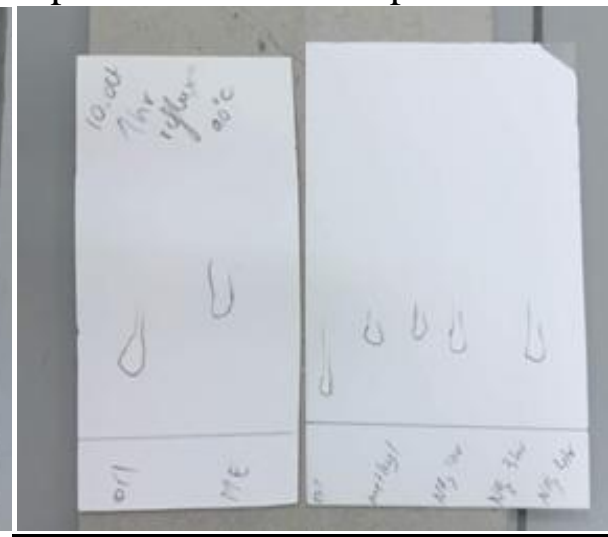

Fig. 8: TLC plates of sample 


\subsection{Fourier Transform Infrared (FTIR)}

As mentioned previously, the spectrum of the vegetable oil is characterized bythe carbonyl group $\mathrm{C}=$ Oabsorption between $1750-1745 \mathrm{~cm}^{-1}$, whereas a fatty acid amide spectrum is characterized by the carbonyl group $-\mathrm{C}=\mathrm{O}$ absorption at $1630-1690 \mathrm{~cm}^{-1}$. Figure 9 below shows a typical example of the FTIR spectra obtained. The first spectrum of an almond oil sample shows the $-\mathrm{C}=\mathrm{O}$ absorption peak at $1743 \mathrm{~cm}^{-1}$. Comparing this spectrum that was mentioned earlier, it specifies that the sample examined belongs to the triglycerides of oil. The second spectrum shows the $-\mathrm{C}=\mathrm{O}$ at an absorption frequency of $1741 \mathrm{~cm}^{-1}$ which signifies a functional group transformation from a triglyceride (at $1743 \mathrm{~cm}^{-1}$ ) to a methylester (at $1741 \mathrm{~cm}^{-1}$ ). Comparing the third spectrum which belongs to the sample investigated after 3 hours reflux with ammonia with the first two spectra, it is characterized with the appearance of a different $-\mathrm{C}=\mathrm{O}$ peak at $1644 \mathrm{~cm}^{-1}$ which falls in the range of an amide carbonyl group (1630-1690 $\left.\mathrm{cm}^{-1}\right)$ indicating a functional group transformation and the formation of the desired fatty acid amide. Note that the intensity of peaks rarely correlate to concentration.

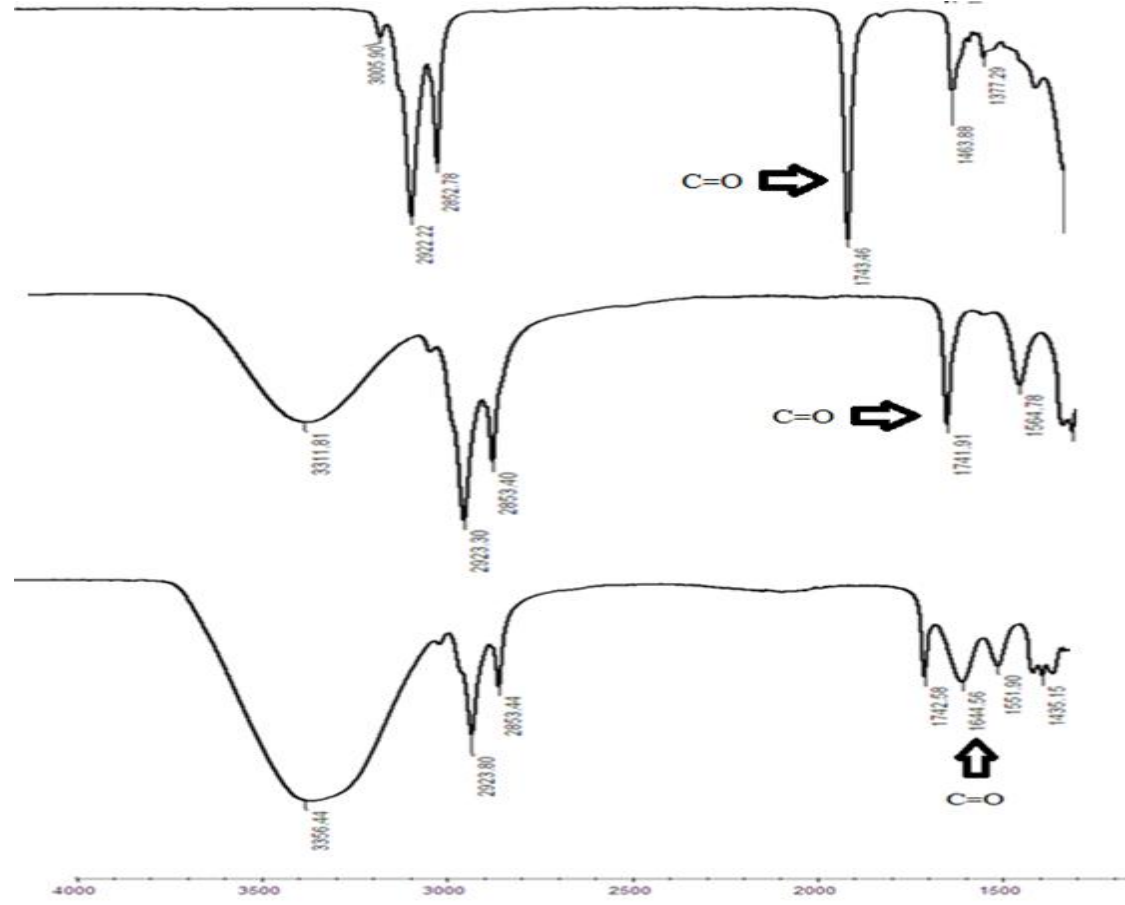

Fig. 9: FTIR spectra of the almond sample

\subsection{Linear Polarization Resistance (LPR)}

The inhibition effectiveness of the amide produced was measured by repeated LPR measurement. LPR scans were repeated every 5 minutes for 2 hours and plotted versus time. This helps to show correlated LPR data. A typical example of the plot of the LPR measurements is shown in Figure 10 below which represents the almond amide sample. The two graphs that are plotted show the corrosion rate vs. time of the almond sample measured by LPR for both the uninhibited and the inhibited samples.

A difference in the average corrosion rate between the uninhibited (light grey) and inhibited (dark grey) sample is shown. A change in the average corrosion rate from $1.4 \mathrm{~mm} / \mathrm{yr}$ before injection to $0.032 \mathrm{~mm} / \mathrm{yr}$ after injection of the almond amide to the system indicates the inhibition efficiency of the amide synthesized.

Results of the inhibition efficiency and reduction ratio of all the samples investigated are summarized in Table 1. The inhibition efficiency varies between the lowest percentages to the highest from 58 to $98 \%$ based on the sample. Canola oil showed a very low (58\%) inhibition efficiency compared to the other oil samples.

The efficiency was calculated:

$$
\% \text { Efficiency }=\frac{\text { Corr }_{\text {uninhibited }}-\text { Corr }_{\text {inhibited }}}{\text { Corr }_{\text {uninhibited }}} \times 100
$$

Thus for almond oil:

$$
\% \text { Efficiency }=\frac{0.70-0.43}{0.43} x 100=62.8 \% \text {; Alternatively, ratio }=\frac{0.7}{0.43}=1.62
$$




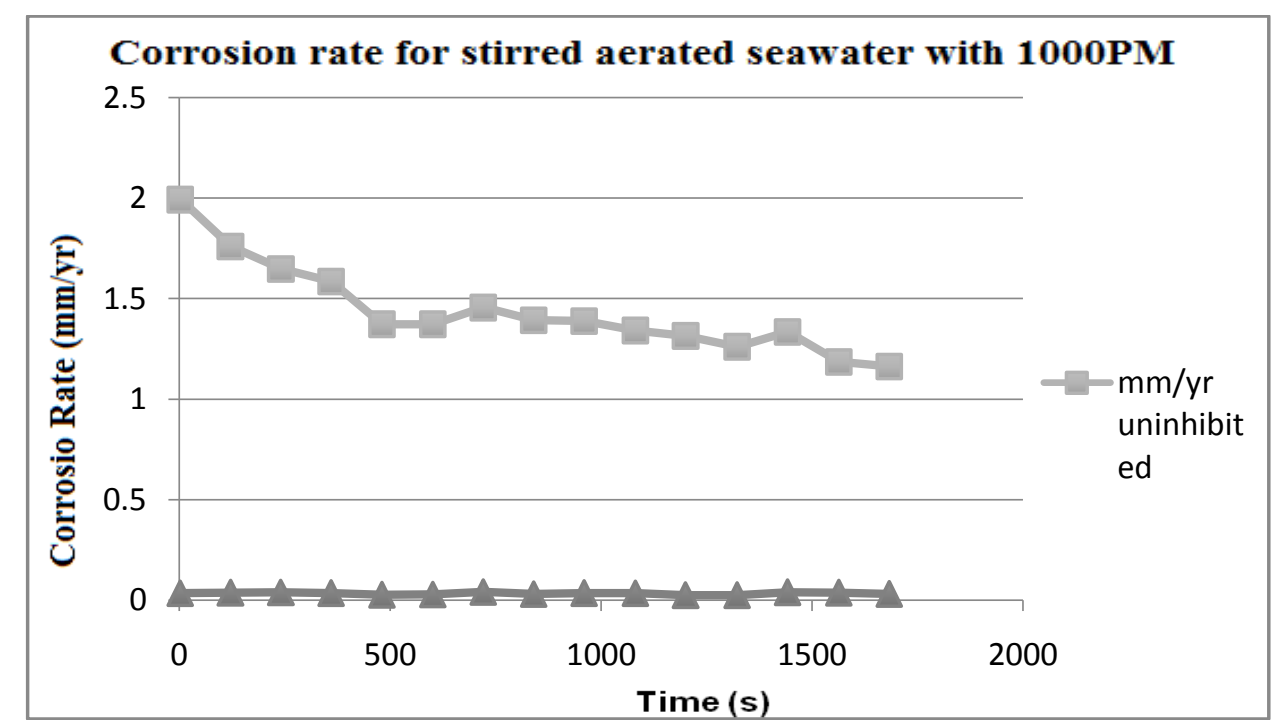

Fig. 10: Corrosion rate vs. time of almond sample

Table 1: LPR corrosion efficiency measurements of the six types of vegetable oil.

\begin{tabular}{|l|l|l|l|l|l|}
\hline Sample & & $\begin{array}{l}\text { Uninhibited corrosion } \\
\text { rate }(\mathbf{m m} / \mathbf{y r})\end{array}$ & $\begin{array}{l}\text { Inhibited corrosion } \\
\text { rate }(\mathbf{m m} / \mathbf{y r})\end{array}$ & $\begin{array}{l}\text { Inhibitor } \\
\text { Efficiency }(\boldsymbol{\%})\end{array}$ & $\begin{array}{l}\text { Reduction } \\
\text { Ratio }\end{array}$ \\
\hline \multirow{3}{*}{ IImond } & Oil & $0.43+/-0.09$ & $0.7+/-0.1$ & -0.6 & 0.06 \\
& $\mathbf{M E}$ & $1.00+/-0.07$ & $0.50+/-0.08$ & 50 & 2.00 \\
& Amide & $1.4+/-0.2$ & $0.032+/-0.005$ & 98 & 43.0 \\
\hline \multirow{2}{*}{ Cress } & Oil & $0.6+/-0.2$ & $0.3+/-0.2$ & 50 & 1.00 \\
\cline { 2 - 6 } & Amide & $1.8+/-0.4$ & $0.18+/-0.03$ & 90 & 10.0 \\
\hline \multirow{3}{*}{ Linseed } & Oil & $1.00+/-0.2$ & $0.9+/-0.1$ & 10 & 1.00 \\
\cline { 2 - 6 } & ME & $0.4+/-0.2$ & $0.160+/-0.007$ & 60 & 3.00 \\
\cline { 2 - 6 } & Amide & $1.0+/-0.2$ & $0.038+/-0.003$ & 96 & 29.0 \\
\hline \multirow{2}{*}{ Peanut } & Oil & $1.00+/-0.04$ & $0.7+/-0.1$ & 30 & 0.50 \\
\cline { 2 - 6 } & Amide & $1.5+/-0.1$ & $0.18+/-0.04$ & 88 & 13.0 \\
\hline \multirow{2}{*}{ Sunflower } & Oil & $0.8+/-0.2$ & $1.6+/-0.8$ & -1.0 & 0.50 \\
\cline { 2 - 6 } & Amide & $1.0+/-0.3$ & $0.09+/-0.03$ & 91 & 13.0 \\
\hline Canola & Amide & $\mathbf{0 . 7 3 + / - 0 . 0 6}$ & $\mathbf{0 . 3 1 + / - 0 . 0 2}$ & $\mathbf{5 8}$ & $\mathbf{2 . 0 0}$ \\
\hline
\end{tabular}

\subsection{Mass Loss}

The corrosion inhibition efficiency and reduction ratio measured by mass loss were investigated and showed correlation with the results obtained by LPR. The mass loss is calculated using the equation below:

MassLoss $=\frac{\Delta \text { weight }}{\text { area }}$

For instance, for the control at $1000 \mathrm{ppm}$,

MassLoss $=\frac{17.906-17.118}{2.7}=0.291 \mathrm{gcm}^{-2}$

The calculation of the corrosion rate was done using the equation below:

Rate $=\frac{K W}{A T D}=\frac{8740 \times 0.291}{168 \times 7.87}=1.92 \mathrm{mmyr}^{-1}$

Where

$\mathrm{K}=8.74 \times 10^{4} \mathrm{~mm} / \mathrm{yr}, \mathrm{T}=168 \mathrm{hrs}, \mathrm{d}=7.87 \mathrm{~g} / \mathrm{cm}^{3}$, 
Table 2 below shows the mass loss, corrosion rates, corrosion inhibition efficiency and reduction rate for all the amide samples. The corrosion efficiency vary between 86 and $95 \%$ depending on the different amide used and correlate with the LPR corrosion efficiencies which vary between 88 and $98 \%$.

Table 2: Corrosion inhibition efficiency and of the six types of vegetable oil.

\begin{tabular}{|l|l|l|l|l|l|l|}
\hline Samples & $\begin{array}{l}\text { Inhibitor } \\
\text { Amount } \\
(\mathrm{ppm})\end{array}$ & $\begin{array}{l}\text { Mass loss } \\
\left(\mathrm{g} / \mathrm{cm}^{2}\right)\end{array}$ & Time (hrs) & $\begin{array}{l}\text { Corrosion } \\
\text { rate } \\
(\mathrm{mm} / \mathrm{yr})\end{array}$ & $\begin{array}{l}\text { Corrosion } \\
\text { efficiency } \\
(\%)\end{array}$ & $\begin{array}{l}\text { Corrosion } \\
\text { rate } \\
\text { reduction }\end{array}$ \\
\hline Control & 0 & 0.291 & 168 & 1.92 & 0.0 & 1.0 \\
\hline Almond & 1000 & 0.023 & 168 & 0.152 & 92 & 13 \\
\hline Cress & 1000 & 0.023 & 168 & 0.152 & 92 & 13 \\
\hline Linseed & 1000 & 0.016 & 168 & 0.106 & 95 & 18 \\
\hline Peanut & 1000 & 0.0408 & 168 & 0.269 & 86 & 7.0 \\
\hline Sunflower & 1000 & 0.018 & 168 & 0.119 & 94 & 16 \\
\hline
\end{tabular}

\section{Conclusions}

The product of our synthesis experiments without any cleanup or purification, showed great efficacy as corrosion inhibitors with corrosion efficiencies of up to $98 \%$ which correlates with the corrosion rate reduction ratios of up to 44.

Some vegetable oils were more effective as corrosion inhibitors than others. This could possibly be due to the different types of fatty acids present and their different geometries. If we look at the typical composition data, the best vegetable oils were those containing unsaturated acids where the molecule is not a straight chain of hydrocarbon.

In general, the structural configuration of fatty acids is divided into two types; the saturated and the unsaturated configuration. The main difference between them is the presence or absence of double bonds in their chains. The absence of double bond in the saturated fatty acids such as stearic acid, shown in Figure 11 below, allows them to be stacked together which results in more close intermolecular interactions with other molecules. However, the presence of one or more double bond in unsaturated fatty acids such as the linoleic acid, leads to more bends in the molecule due to the cis configuration. These molecules do not stack and the intermolecular interactions between them are much weaker. The chain becomes more bent and less free to interact with other fatty acid molecules and will be therefore more available to stick on the metal surface to prevent against corrosion [24-27].

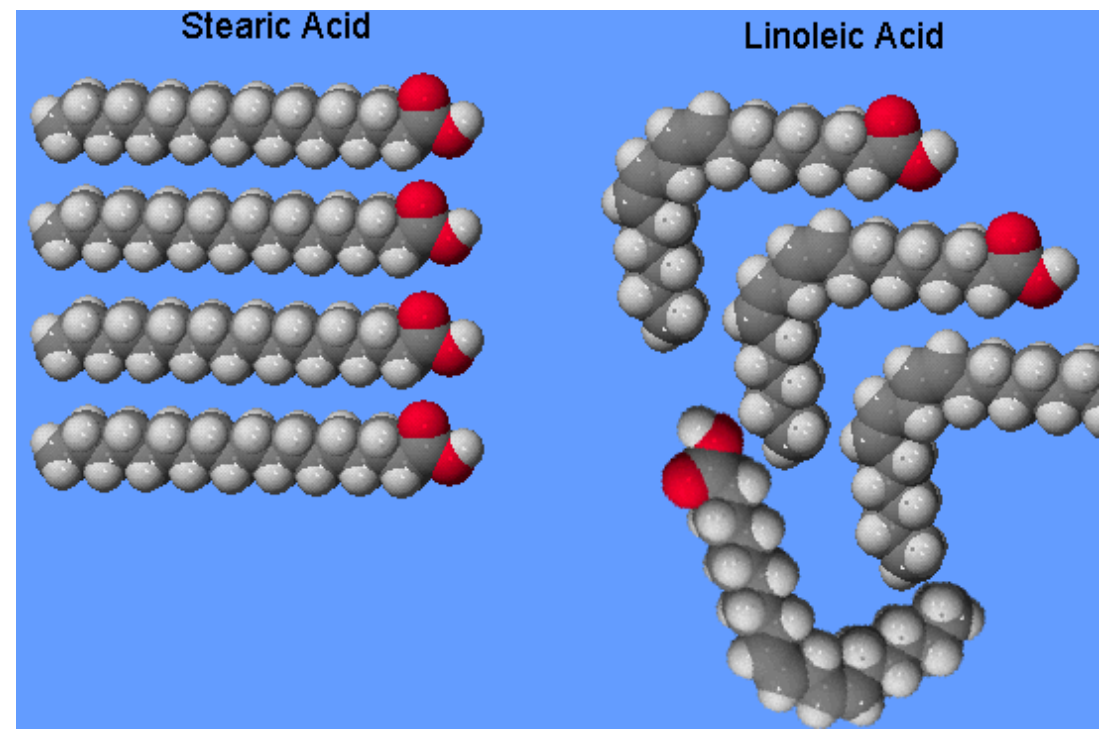

Fig. 11: Chemical structure of stearic and linoleic acid. 
Acknowledgements-The authors acknowledge and appreciate the role of the College of Arts and Sciences at the Petroleum Institute Khalifa University for Science and Technology, for general funding and provision of facilities for this research.

\section{References}

1. Ahmad Z., Principles of corrosion engineering and corrosion control, Butterworth-Heinemann, ISBN-13: 978-0750659246, (2006) 68.

2. Uhlig H., Corrosion and Corrosion Control, J. Wiley ISBN-13:978-0471732792, (1971) 56.

3. Shreir B., G Corrosion, Newnes, ISBN-13:978-0750610773, (2013) 25

4. Jones D. A., Principles and prevention of corrosion, Prentice Hall ISBN -13:978013-359993, (1996) 3, 11, 17, 247.

5. Rostron P., Kasshanna S., International Journal of Corrosion, (2015) ID 851698. http://dx.doi.org/10.1155/2015/851698

6. Flenner P., Carbon Steel Handbook, EPRI (2007) 15.

7. Honeycombe R. W. K., Steels Edward Arnold Ltd (ASIN B01JXMT414) (1981) 75.

8. Shreir B. G., Corrosion, 1, Newnes-Butterworth, ISBN-13:978-0750610773, (1976) 55.

9. Garverick L., Corrosion in the Petrochemical Industry ASM international. ISBN-13:978-0871705051, (1994) 214

10. Shreir B. G., Corrosion, vol 1, Butterworth-Heinemann, ISBN-13:978-0750610773, (1994) 44.

11. Koch G. H., Historic congressional study: Corrosion costs and preventative strategies in the United States, Library of Congress (2002).

12. Sastri E. M., Corrosion prevention and protection: practical solutions, John Wiley (2007) 45.

13. Sabasi M., J. Electrochem. Soc. 8 (2012) 364.

14. Chaudhari G. V., Prog. Org. Coatings, 76 (2013) 1779.

15. Toscano D. D., Biomass and bioenergy, 46 (2012) 511.

16. Singh S. D., Renewable and Sus. Ennergy. Revs, 14 (2010) 200.

17. Carey F. R., Organic Chemistry, McGraw-Hill ISBN-13:978-0077354770, (2002) 256.

18. Al-Jasser M. S., Sc. Wrld. J., 2012 (2012) 5.

19. Yoo S., Corr. Sci., 59 (2012) $42-54$

20. Pramanik K. N., Ind. Eng. Chem. Res., 52 (2013) 5700.

21. Elkadi K. M. Br. Biotech. J., 3 (2013) 500.

22. Abel-Anyebe E. A., Int. J. Chem. 5(1) (2013) 80.

23. Schuchardta U., J. Brazil. Chem. Soc., 9 (1997).

24. Rekkab S., Zarrok H., Salghi R., Zarrouk A., Bazzi Lh., Hammouti B., Kabouche Z., Touzani R., Zougagh M., J. Mater. Environ. Sci. 3 (4) (2012) 613-627

25. Nielsen J.C., Horn N.S., Food Enrichment with Omega-3 Fatty Acids. Elsevier. ISBN-13:978-0857094285, (2013) 5

26. Afia L., Salghi R., Bammou L., Bazzi El., Hammouti B., Bazzi L., Bouyanzer A., Journal of Saudi Chemical Society, 18 (2014) 19-25

27. Saufi H., Al Maofari A., El Yadini A., Eddaif L., Harhar H., Gharby S., El Hajjaji S., J. Mater. Environ. Sci., 6 (2015) 1845-1849

(2017) ; http://www.jmaterenvironsci.com 